\title{
ENGINE: a Phase III randomized placebo controlled study of enzastaurin/R-CHOP as frontline therapy in high-risk diffuse large B-cell lymphoma patients with the genomic biomarker DGM1
}

Grzegorz S Nowakowski ${ }^{1}$, Jun Zhu*,2, Qingyuan Zhang ${ }^{3}$, Joshua Brody ${ }^{4}$, Xiuhua Sun ${ }^{5}$, Joseph Maly ${ }^{6}$, Yuqin Song ${ }^{2}$, Syed Rizvi ${ }^{7}$, Yongping Song ${ }^{8}$, Frederick Lansigan ${ }^{9}$, Hongmei Jing ${ }^{10}$, Junning Cao ${ }^{11}$, Jennifer K Lue ${ }^{12}$, Wen Luo ${ }^{13}$, Lei Zhang ${ }^{13}$, Ling Li ${ }^{13}$, Isabel Han ${ }^{13}$, Joan Sun ${ }^{13}$, Manoj Jivani ${ }^{13}$, Young Liu' ${ }^{13}$, Thomas Heineman ${ }^{13}$ \& Stephen D Smith ${ }^{14}$

${ }^{1}$ Division of Hematology, Mayo Clinic, Rochester, MN, USA

${ }^{2}$ Peking University Cancer Hospital \& Institute, Beijing, PR China

${ }^{3}$ Harbin Medical University Cancer Hospital, Harbin, PR China

${ }^{4}$ Icahn School of Medicine at Mount Sinai, New York, NY, USA

${ }^{5}$ The Second Hospital of Dalian Medical University, Dalian, PR China

${ }^{6}$ Norton Cancer Institute, Louisville, KY, USA

${ }^{7}$ University of Texas Southwestern Medical Center, Dallas, TX, USA

${ }^{8}$ Affililiated Cancer Hospital of Zhengzhou University, Zhengzhou, PR China

${ }^{9}$ Dartmouth-Hitchcock Medical Center, Lebanon, NH

${ }^{10}$ Peking University Third Hospital, Beijing, PR China

${ }^{11}$ Fudan University Shanghai Cancer Center, Shanghai, PR China

${ }^{12}$ Columbia University Medical Center, New York, NY, USA

${ }^{13}$ Denovo Biopharma LLC, San Diego, CA, USA

${ }^{14}$ University of Washington/Fred Hutchinson Cancer Center, Seattle, WA, USA

*Author for correspondence: Tel.: +86 108819 6109; zhu-jun2017@outlook.com

While combination of rituximab, cyclophosphamide, doxorubicin, vincristine and prednisone (R-CHOP) cures most patients with diffuse large B-cell lymphoma (DLBCL), those with high-risk international prognostic index disease have inferior survival. Enzastaurin as a potent inhibitor of PKC- $\beta$ and PI3K/AKT pathway suppressor has been tested in many clinical trials including two key studies in DLBCL: Phase III maintenance study (Preventing Relapse in Lymphoma Using Daily Enzastaurin [PRELUDE]) and a first-line Phase II study (S028). DNA extracted from PRELUDE patients' blood samples was retrospectively genotyped identifying a novel genetic biomarker, DGM1 that showed high correlation with response to enzastaurin. A similar finding observed in the S028 study suggested that addition of enzastaurin to R-CHOP may significantly improve outcomes as frontline therapy for high-risk DGM1 positive DLBCL patients. ENGINE is a global, multicenter, placebo-controlled and randomized study to compare the effect of R-CHOP/enzastaurin as frontline treatment in high-risk DLBCL patients. The primary end point for this study is overall survival in patients who are DGM1 positive. Clinical Trial Registration Identifier: NCT03263026

First draft submitted: 28 February 2020; Accepted for publication: 24 March 2020; Published online: 6 April 2020

Keywords: DGM1 • enzastaurin $\bullet$ lymphoma $\bullet$ personalized chemotherapy

Diffuse large B-cell lymphoma (DLBCL) is a heterogeneous and aggressive tumor that affects mainly adults in their 60s-70s. This B-cell lymphoid malignancy is the most common histologic subtype of non-Hodgkin lymphoma [1-5] with an incidence of nearly $25-40 \%$ of all new cases of non-Hodgkin lymphoma diagnosed worldwide. Additionally, increases in the incidence of DLBCL have recently been reported in Central and South America as well as in Asia [3,4]. 
Despite the evaluation of a number of other agents in randomized trials, combination of rituximab, cyclophosphamide, doxorubicin, vincristine and prednisone (R-CHOP) administered every 21 days has remained the standard of care for all DLBCL subtypes for over 2 decades. While R-CHOP cures over $60 \%$ of DLBCL patients, 5 -year survival rates vary from $90 \%$ for low-risk DLBCL patients to less than $40-50 \%$ for high-risk DLBCL patients as defined by the international prognostic index (IPI) score [4-9]. This heterogeneity is not well understood, and there is an urgent need to improve R-CHOP therapy, with one possible method being adding strategic agents to this backbone as an up-front option for high-risk patients [8]. Substantial effort has been devoted to developing clinical models and molecular and gene expression profiling as tools to identify DLBCL patients with comparatively inferior prognosis to R-CHOP therapy in order to determine which patients are the most suitable candidates for novel targeted therapies $[4-8,10,11]$.

Analysis of samples from high-risk IPI patients that failed R-CHOP, identified PKC- $\beta$ as a potential therapeutic target in this group [12-14]. PKC- $\beta$ belongs to the PKC family known for their important regulatory functions within normal cells. The PKC family has also been a focus of research due to its role in the pathophysiology of cancer, such as promoting and/or inhibiting tumor growth, differentiation, tumor-associated angiogenesis and inhibition of apoptosis [11-14]. The contribution of the different PKC isozymes in cancer growth or suppression is variable [15,16]. In particular, PKC- $\beta$ is a component of the B-cell receptor in both normal and clonal B-lymphocytes, and it is associated with tumor-stimulated angiogenesis upon its regulation of the VEGF pathway [16] and also with survival of these clones. High levels of PKC- $\beta$ are associated with an inferior prognosis in some specific DLBCL subtypes making it a potential therapeutic target [5,13-18].

\section{Enzastaurin}

The role of PKC- $\beta$ in the proliferation and survival of clonal B-lymphocytes led to the development of selective drugs targeting this regulatory pathway. Similar to other protein kinases, PKC consists of a catalytic and a regulatory region, and the enzyme structure is linked to their activation through molecules such as diacylglycerol (DAG) alone or DAG and calcium. In general, PKC inhibitors can be classified into three broad categories: PKC inhibitors that interfere with the binding site of second messengers and DAG; molecules that prevent the binding of receptor for activated C kinase and lastly; ATP competitive inhibitors that prevent activation of PKC enzymes [19].

Enzastaurin is an acyclic bisindolylmaleimide and an oral formulated selective serine-threonine ATP-competitive inhibitor of PKC- $\beta$ kinase and the cell cycle regulatory signaling transduction pathway PI3K/AKT [19]. Enzastaurin inhibits the phosphorylation of downstream signal proteins associated with tumor suppression and induced angiogenesis [19]. The antitumor activity of enzastaurin has been demonstrated in vitro and in various cancer models including gastric and breast cancers, brain tumors and DLBCL [12,15-20].

Enzastaurin was developed by Eli Lilly and Company, IN, USA (Lilly) and evaluated in 51 clinical studies and 15 clinical pharmacology studies with 39 single-agent studies (enzastaurin alone or enzastaurin combined with non-neoplastic agents) and 27 combination studies (enzastaurin plus another anticancer agent). As of September 2013, among the patients exposed to enzastaurin, 3149 were cancer patients and 188 were healthy individuals. Lilly's enzastaurin studies in DLBCL patients are summarized in Table 1 below.

Based on several Phase I studies Lilly conducted the recommended Phase II dose of enzastaurin in DLBCL was $500 \mathrm{mg}$. The safety and tolerability of enzastaurin $500 \mathrm{mg}$ was confirmed in subsequent Phase II studies, with no serious adverse grade $3 / 4$ effects or drug-associated deaths [15,17,21].

Pharmacology studies have proved that enzastaurin is primarily metabolized in the liver with minimal renal elimination. When enzastaurin was studied in cancer patients with hepatic impairment, drug exposures tended to be lower compared with patients with normal hepatic function and decreased with increasing severity of hepatic dysfunction in previous studies. Thus, it is not recommended to administer enzastaurin to patients with moderate or severe hepatic impairment.

In addition, enzastaurin is metabolized primarily by CYP4503A (CYP3A) as demonstrated in the H6Q-MCJCAJ Phase II trial using enzastaurin alone in patients diagnosed with recurrent high-grade glioma [16]. Patients on enzyme-inducing anti-epileptic drugs such as phenytoin and carbamazepine had significantly decreased enzastaurin exposures. Thus, concomitant medications that are potent inducers or inhibitors of isozyme CYP3A affect enzastaurin exposure.

Despite the favorable and promising preclinical antitumor activity of enzastaurin in several cancer subtypes, it has been very difficult to replicate these results in clinical trials. Enzastaurin was evaluated as single agent as well as in combination with various chemotherapy regimens in several clinical trials, all of which have failed to support a 
Table 1. Summary of enzastaurin studies in diffuse large B-cell lymphoma.

\begin{tabular}{|c|c|c|}
\hline Study ID/title & Patients (n) & Ref. \\
\hline $\begin{array}{l}\text { H6Q-MC-JCAI } \\
\text { A multicenter, nonrandomized, open-label, single arm, Phase II study of enzastaurin in patients with } \\
\text { relapsed/refractory DLBCL }\end{array}$ & 55 & [18] \\
\hline $\begin{array}{l}\text { H6Q-M-S013 } \\
\text { Open-label, single-arm, Phase II study of rituximab, gemcitabine and oxaliplatin plus oral enzastaurin as treatment } \\
\text { for patients with relapsed DLBCL }\end{array}$ & 68 & [18] \\
\hline $\begin{array}{l}\text { H6Q-MC-S028 } \\
\text { Open-label, randomized, Phase II study of R-CHOP plus enzastaurin vs R-CHOP in the first-line treatment of patients } \\
\text { with intermediate and high-risk diffuse large B-cell lymphoma }\end{array}$ & $\begin{array}{l}101 \text { (100 evaluable) } \\
57 \text { enzastaurin } \\
43 \text { placebo }\end{array}$ & [18] \\
\hline $\begin{array}{l}\text { H6Q-MC-JCBJ } \\
\text { A Phase III study to investigate the prevention of relapse in lymphoma using daily enzastaurin }\end{array}$ & $\begin{array}{l}758 \text { randomized } \\
742 \text { treated } \\
\text { Enzastaurin } 493 \\
\text { Placebo } 249\end{array}$ & [17] \\
\hline
\end{tabular}

DLBCL: Diffuse large B-cell lymphoma; R-CHOP: Rituximab, cyclophosphamide, doxorubicin, vincristine and prednisone.

statistically and clinically significant beneficial effect of enzastaurin, with the exception of in DLBCL. Specifically, in a Phase II study of enzastaurin administered as a single agent in patients with relapsed or refractory DLBCL (n = 55) [10], four patients (7\%; 95\% CI: 2-18\%), including three complete responders and one patient with stable disease, experienced free from progression ranging 20-60 months after study entry. This led to the development of a Phase III clinical trial entitled: 'Preventing Relapse in Lymphoma Using Daily Enzastaurin' (PRELUDE) [17].

The PRELUDE study [17] compared disease-free survival (DFS) after maintenance therapy with enzastaurin versus placebo in patients with DLBCL in complete response (CR) and with a high risk of relapse after first-line therapy. This was a multinational, double blind, randomized, placebo-controlled study that enrolled 758 high-risk DLBCL (IPI $\geq 3$ ) patients in CR or complete response unconfirmed and/or with a negative fluorodeoxyglucosePET scan post completion of six to eight cycles of R-CHOP. Patients were randomly selected in a 2:1 ratio to receive either enzastaurin $500 \mathrm{mg}$ daily or an identical placebo as maintenance for 3 years. The primary end point was DFS defined as lack of disease progression or death. The data collected was analyzed 36 months after the last enrolled patient initiated treatment [17]. The DFS hazard ratio (HR) for enzastaurin compared with placebo was 0.92 (95\% CI: $0.69-1.22$; 2-sided log-rank p = 0.54). The DFS at 24 and 48 months were 78 and $70 \%$ for the enzastaurin arm, and 75 and $71 \%$ for placebo, respectively [17]. The overall survival (OS) at 24 and 48 months was 87 and $81 \%$ for enzastaurin, and 89 and $82 \%$ for placebo [17]. No difference was found in 4-year DFS (70 vs $71 \%$ ) or OS (81 vs $82 \%)$ between the enzastaurin and placebo arms, demonstrating the lack of benefit of enzastaurin when given only as maintenance in previously untreated DLBCL [17].

The benefit of enzastaurin concurrent with R-CHOP, versus R-CHOP alone, in previously untreated DLBCL was then evaluated in the randomized Phase II study $(\mathrm{S} 028, \mathrm{n}=101)$ [20]. In this trial, 101 patients were randomized $3: 2$ to receive $\mathrm{R}-\mathrm{CHOP} /$ enzastaurin $(\mathrm{n}=58)$ or $\mathrm{R}-\mathrm{CHOP}(\mathrm{n}=43)$ as first-line therapy for intermediate or high-risk DLBCL. Following the initial induction treatment period, patients in the enzastaurin group with CR or partial response (PR) also continued to receive enzastaurin for up to 3 years or until disease progression. The median progression-free survival (PFS) for patients in the enzastaurin arm was 36.2 months versus 22.6 months in the control arm $(\mathrm{HR}=0.73 ; \mathrm{p}=0.151)$. After adjusting for imbalances in baseline IPI score $(\leq 2 \mathrm{vs}>2)$ and Eastern Cooperative Oncology Group performance status ( 0 vs 1 or 2), the HR was 0.59 (95\% CI: 0.32-1.09; one sided $\mathrm{p}=0.048$ ). In a post-hoc subgroup analysis, patients with an IPI score $\geq 3$ had a median PFS that was not evaluable in the enzastaurin arm compared with 8.9 months in the control arm $(\mathrm{HR}=0.50 ; \mathrm{p}=0.037)$. The 2-year OS was 75 and $69 \%$ in the overall population, and 71 and $55 \%$ in IPI $\geq 3$ patients $(\mathrm{p}=0.186)$ [20].

While these studies failed to meet their primary end point, the S028 trial data established the tolerability of concurrent R-CHOP/enzastaurin and the improved PFS in high-risk IPI patients after treatment with this combination. Collectively, these data stimulated further efforts to identify a pretreatment biomarker capable of predicting patients who benefit most from this combination. 


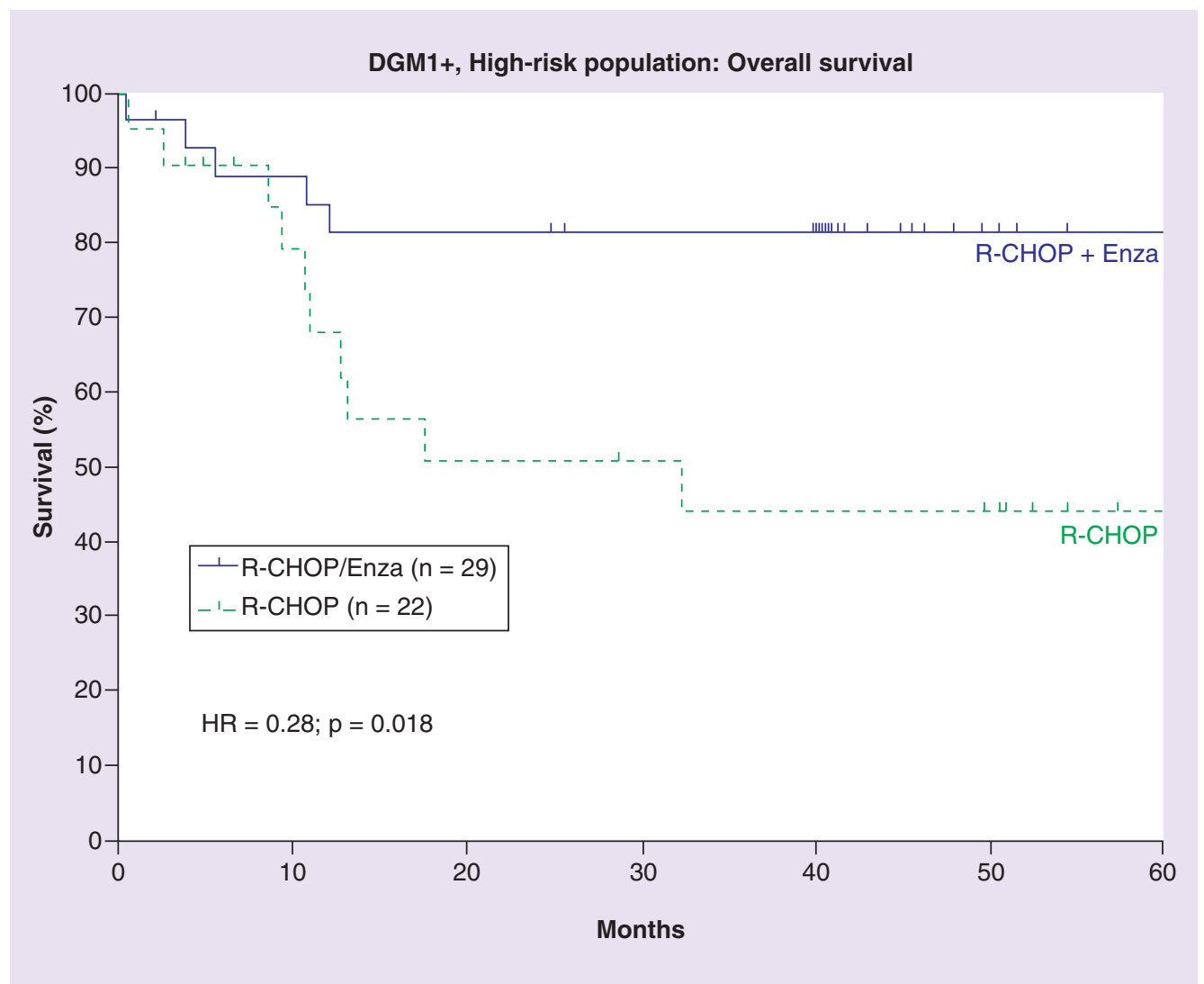

Figure 1. Overall survival on RCHOP+enzastaruin vs R-CHOP alone in high-risk DGM 1+ patients for S028 study. R-CHOP: Rituximab, cyclophosphamide, doxorubicin, vincristine and prednisone.

\section{Discovery \& validation of DGM1}

Even though enzastaurin has failed to demonstrate efficacy in overall population in PRELUDE and S028 trials [22,23], there were signs that there might be subsets of patients who might be benefited from the enzastaurin treatment. Another challenge is that there are no biopsies, which are typically required to study biomarkers for oncology drug, available for most of the patients enrolled in these studies. However, there are evidences suggesting that germline polymorphism could be biomarkers for cancer drug, such BRCA germline variation has been proved to be predicator for olaparib. Thus, DNA samples extracted from blood of patients (high risk IPI $>=3$ ) enrolled in the PRELUDE study were genotyped using whole genome single nucleotide polymorphism (SNP) arrays and approximately 5 million SNPs were analyzed. It was found that a specific configuration of two SNPs (rs309605 and rs309604) located on chromosome 8 was strongly associated with survival in the enzastaurin arm. The p-value for this association was $5.8 \times 10^{-9}$. To put this in context, a p-value less than $5 \times 10^{-8}$ is generally suggestive of genome-wide significance. This biomarker is now referred to as DGM1. The closest gene to DGM1 is TRPS1, which encodes transcriptional repressor GATA binding 1 or TRPS1 protein. TRPS1 represses GATA-regulated genes and plays a central role in cell cycle control and tumor development, which may explain partially why it may be related to enzastaurin activity [24,25].

To confirm these findings from the PRELUDE trial, an analysis of the DGM1 biomarker was performed using archived DNA samples from patients enrolled in the S028 study. This investigation demonstrated significant improvement in OS (HR: 0.28; $\mathrm{p}=0.018$ ) for high-risk (IPI $\geq 3$ ) DGM1+ patients receiving R-CHOP plus enzastaurin compared with high-risk DLBCL DGM1+ patients receiving R-CHOP alone (Figure 1). These results suggest that addition of enzastaurin to R-CHOP may significantly improve outcome in frontline high-risk DGM1+ DLCBL patients [18].

DGM1 was additionally evaluated in control arm patients who were treated with R-CHOP alone in S028 study. Results indicated that DGM1 status was not predictive of efficacy in the control (R-CHOP only) arm arguing 


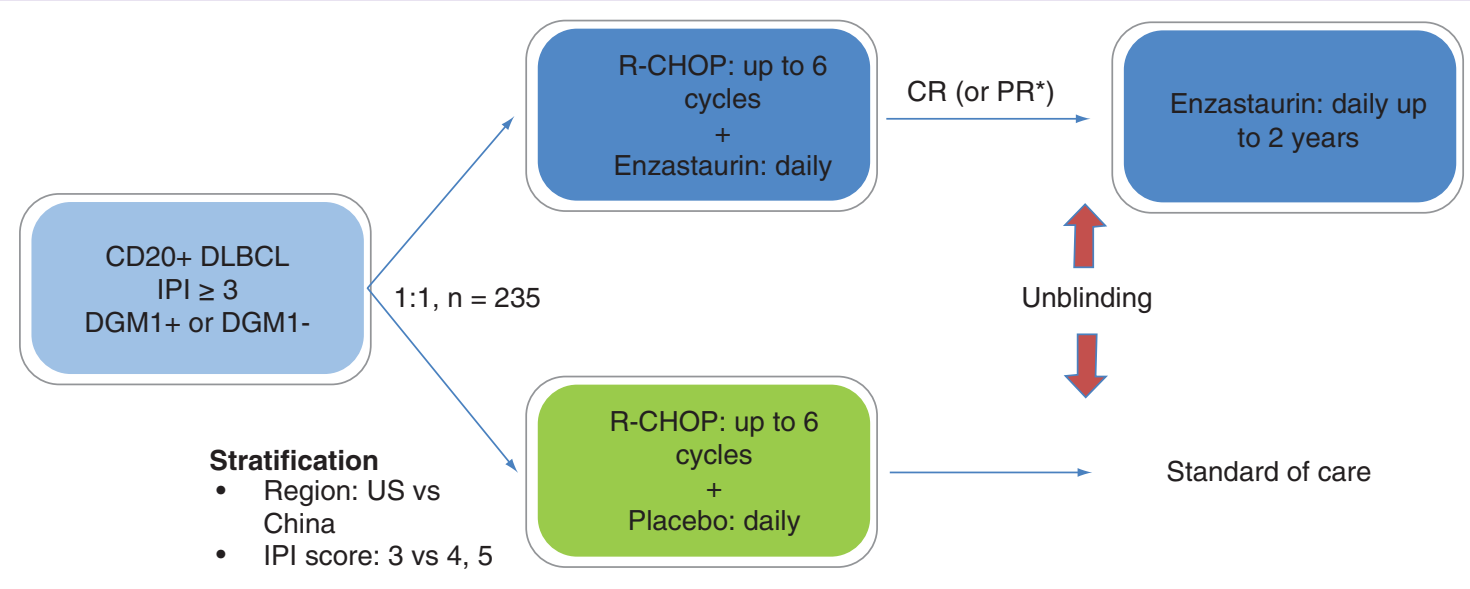

Figure 2. ENGINE study design.

R-CHOP: Rituximab, cyclophosphamide, doxorubicin, vincristine and prednisone.

against DGM1 as a prognostic biomarker. Similar result was observed in control arm patients who received placebo in Phase III PRELUDE trial.

\section{ENGINE study}

\section{Study rationale}

The Phase III ENGINE study was designed to prospectively validate the DGM1 biomarker analysis results from the Phase II study (S028) in patients with newly diagnosed high-risk DLBCL. The primary objective is the effect of adding enzastaurin to R-CHOP therapy in high-risk IPI $\geq 3$ DLBCL patients who possess the DGM1 biomarker on OS. The study aims to determine whether there is markedly improved survival in this enriched population compared with the current standard of care, R-CHOP alone. This study is being conducted in the USA and China. The prevalence of biomarker-positive patients ranges between approximately $68 \%$ for patients of African ancestry living in the Southwest USA, approximately $90 \%$ for patients of European ancestry and approximately $95 \%$ of patients living in Beijing, China (International Genome Sample Resource -1000 Genomes Project).

\section{Study design}

ENGINE is a Phase III, randomized double blind, placebo-controlled, multicenter study in patients with treatment naive high-risk DLBCL. Approximately 235 patients will be randomized in a 1:1 ratio in the USA and China, stratified by IPI score $(3$ vs 4,5$)$ and region (USA vs China; Figure 2). DGM1 biomarker testing is performed in batches retrospectively after randomization.

All patients will receive up to six cycles (one cycle $=21$ days) of treatment during the combination phase. PETcomputed tomography will be used for staging and to assess radiographic response at the end of combination phase treatment. Each patient's treatment assignment will be unblinded after combination phase tumor response assessment. Patients randomized to the enzastaurin arm who achieve a complete response (CR) or PR (at investigator's discretion) by the Lugano Classification (Cheson 2014) will have the opportunity to continue in the single-agent phase of the study and receive single-agent enzastaurin for up to 2 additional years (Figure 2).

\section{Study objectives}

- Primary objective is to compare the effect of R-CHOP plus enzastaurin versus R-CHOP plus placebo on OS in treatment-naive patients with high-risk DLBCL who possess the DGM1 biomarker. Note: Both DGM1+ and DGM1 biomarker negative (DGM1-) patients will be enrolled but primary analysis will include only DGM1+ patients. Sites and sponsor will remain blinded to biomarker status of each patient; 
- Secondary objectives are to compare combination phase CR rate and objective response rate (ORR) in DGM1+ patients; evaluate event-free survival (EFS) in all patients and in DGM1+ patients; determine OS of enzastaurin $+\mathrm{R}-\mathrm{CHOP}$ in DGM1- patients and evaluate the safety profile of enzastaurin + R-CHOP.

Study key eligibility

Key inclusion criteria

- $\geq 18$ years;

- Histologically confirmed CD20 positive DLBCL (MYC \& BCL2 and/or BCL6 rearrangements eligible);

- Eastern Cooperative Oncology Group performance status 0, 1 or 2;

- IPI score $\geq 3$;

- DGM1+ or DGM1 -;

- Left ventricular ejection (LVEF) $\geq 50 \%$ by echo or multiple gated acquisition (MUGA);

- Adequate organ function:

- Total bilirubin $\leq 1.5 \times$ upper limit of normal (ULN; $\leq 5 \times$ ULN in the case of Gilberts syndrome, liver or pancreatic involvement by lymphoma);

- $\quad$ ALT \& AST $\leq 2.5 \times$ ULN ( $\leq 5 \times$ ULN if liver involvement);

- Creatinine clearance $\geq 40 \mathrm{ml} / \mathrm{min}$ by Cockcroft-Gault equation;

- $\quad$ Platelet $\geq 75 \times 10^{9} / 1$ ( $\geq 50 \times 10^{9}$ if BM involvement);

- $\quad \mathrm{Hgb} \geq 8 \mathrm{~g} / \mathrm{dl}(\geq 7 \mathrm{~g} / \mathrm{dl}$ if BM involvement);

- $\quad$ Absolute neutrophil count (ANC) $\geq 1.5 \times 10^{9} / 1\left(\geq 1.0 \times 10^{9}\right.$ if bone marrow [BM] involvement).

Key exclusion criteria

- History of indolent lymphoma or follicular grade $3 \mathrm{~b}$ lymphoma;

- Primary mediastinal (thymic) large B-cell lymphoma; B-cell lymphoma unclassifiable;

- Known CNS involvement or Second primary malignancy (SPM);

- Use of a strong inducer or moderate/strong inhibitor of CYP3A4;

- History of long QT syndrome, QT corrected formula (QTcF) >450 ms (males) or >470 ms (females);

- Use of medication that can prolong QT/QTc;

- Ongoing > G2 peripheral neuropathy;

- Evidence of chronic hepatitis $\mathrm{C}$ by antibody to hepatitis $\mathrm{C}$ virus (HCV) with HCV-RNA (+);

- Evidence of chronic hepatitis B either HBsAg (+) or HBcAb (+) with hepatitis B virus (HBV)-DNA (+).

Study end points

- Primary end points:

- OS in patients who are DGM1+. OS is defined as the elapsed time from the date of randomization to the date of death from any cause;

- Secondary end points:

- $\quad$ CR in patients who are DGM1+;

- $\quad$ ORR (CR and PR) in patients who are DGM1+;

- $\quad$ OS in patients who are DGM1-;

- $\quad$ EFS including EFS12 and EFS24, for all the patients, and for the patients who are DGM1+.

ENGINE trial versus other Phase III studies in frontline DLBCL

- Unique genomic biomarker;

- High risk patient population IPI = 3, 4, 5;

- Simplified screening procedures to allow quick treatment initiation, feasible to enroll high-risk patients;

- Eligibility based on local pathology diagnosis;

- Local safety labs;

- Primary end point OS with less frequent imaging schedule;

- 2 years of single agent phase after induction phase. 


\section{Statistical considerations}

Efficacy analysis will be conducted on an intent-to-treat basis. The intent-to-treat population is defined as all patients who are randomized into the trial, regardless of whether they received study treatment. Safety analyses will include data from all patients who have received at least one dose of study treatment. The primary end point is OS in the DGM1+ patients. Study patients will be randomized 1:1 to R-CHOP plus enzastaurin or R-CHOP plus placebo, stratified by IPI score (3 vs 4, 5) and by region (USA vs China). This will be an event driven study. In the trial, 66 events are required to provide $90 \%$ power to detect a HR of 0.45 for OS in patients who are DGM1+, based on a stratified log-rank test at one-sided alpha of 0.025 . Statistical significance will be achieved with an estimated HR $\leq 0.62$. It is expected that $15 \%$ of trial patients will be DGM1-. To achieve 66 events in DGM1+ patients, the trial would enroll approximately 235 patients with target 200 being biomarker positive and 35 being biomarker negative. The treatment difference in OS will be compared using the log-rank test stratified by the randomization factors. Secondary end points include CR, ORR and EFS in DGM1+ patients. CR and ORR will be analyzed using Cochran-Mantel-Haenszel method stratified by randomization factors; and EFS will be analyzed using the method for primary end point of OS.

\section{Study status}

ENGINE is a global study, with centers located in both USA and China. The study is currently recruiting with expected accrual completion in June 2020. The trial is sponsored by Denovo Biopharma. More information is available at ClinicalTrials.gov (NCT03263026).

\section{Conclusion}

While R-CHOP is curative for the majority of patients with DLBCL, up to $40 \%$ of high-risk patients will fail first-line therapy. Only a fraction of relapsed/refractory patients are curable with second-line therapy, and the heterogeneity of outcomes is poorly understood. Targeting signaling pathways active in DLBCL remains a promising avenue of research despite negative randomized trials to date. These trials may have failed partly due to the lack of useful predictive biomarkers to identify patients responsive to novel agents. The ENGINE study will use a de novo genomic biomarker DGM1, which was identified from the PRELUDE and S028 studies, and correlate the presence of the DGM1 biomarker with OS in high-risk DLBCL treatment naive patients who receive enzastaurin/R-CHOP as frontline therapy.

Financial \& competing interests disclosure

This ENGINE study is sponsored by Denovo Biopharma. Among all the coauthors, W Luo, L Zhang, L Li, I Han, J Sun, M Jivani, Y Liu and T Heineman are employees of Denovo Biopharma LLC.

The other coauthors are ENGINE study investigators: GS Nowakowski is a physician from Mayo Clinic, Rochester, MN, USA; J Zhu and $Y$ Song are physicians from Peking University Cancer Hospital \& Institute, Beijing, China; Q Zhang is a physician from Harbin Medical University Cancer Hospital, Harbin, China; J Brody is a physician from Icahn School of Medicine at Mount Sinai, NY, USA; X Sun is a physician from The Second Hospital of Dalian Medical University, Dalian, China; J Maly is a physician from Norton Cancer Institute, Louisville, KY, USA; S Rizvi is a physician from University of Texas Southwestern Medical Center, Dallas, TX, USA; Y Song is a physician from Affliliated Cancer Hospital of Zhengzhou University, Zhengzhou, China; F Lansigan is a physician from DartmouthHitchcock Medical Center, Lebanon, NH, USA; H Jing is a physician from Peking University Third Hospital, Beijing, China; J Cao is a physician from Fudan University Shanghai Cancer Center, Shanghai, China; JK Lue is a physician from Columbia University Medical Center, NY, USA and SD Smith is a physician from University of Washington/Fred Hutchinson Cancer Center, Seattle, WA, USA. The coauthors acknowledge that Contract Research Organization support was executed by Covance. The authors have no other relevant affiliations or financial involvement with any organization or entity with a financial interest in or financial conflict with the subject matter or materials discussed in the manuscript apart from those disclosed.

No writing assistance was utilized in the production of this manuscript.

Open access

This work is licensed under the Attribution-NonCommercial-NoDerivatives 4.0 Unported License. To view a copy of this license, visit http://creativecommons.org/licenses/by-nc-nd/4.0/ 
Executive summary

DLBCL

- Combination of rituximab, cyclophosphamide, doxorubicin, vincristine and prednisone (R-CHOP) administered every 21 days has remained the standard of care for all diffuse large B-cell lymphoma (DLBCL) subtypes for over 2 decades with cures over $60 \%$ of DLBCL patients.

- Addition of novel agents to R-CHOP as frontline therapy in high-risk IPI score DLBCL patients is a priority.

Enzastaurin

- Enzastaurin is a selective serine-threonine ATP-competitive inhibitor of PKC- $\beta$ and the regulatory signaling transduction pathway PI3K-AKT that inhibits the phosphorylation of downstream signal proteins associated with tumor suppression and induced angiogenesis with in vitro antitumor activity demonstrated in various cancer models including DLBCL.

- Enzastaurin safety and efficacy has been tested in several clinical trials including two key studies in DLBCL: a Phase III maintenance study (Preventing Relapse in Lymphoma Using Daily Enzastaurin [PRELUDE]) and a first-line Phase II study (S028). These trials may have failed partly due to the lack of useful predictive biomarkers to identify patients responsive to novel agents.

DGM1

- DNA extracted from PRELUDE patients' blood samples was retrospectively genotyped using whole genome single nucleotide polymorphism arrays. A novel genetic biomarker, Denovo genomic marker 1 (DGM1), showed a high correlation with response to enzastaurin, similar to S028 study that demonstrated a HR $0.28(0.1-0.81)$ for OS in high-risk (IPI $\geq 3$ ) DGM1+ patients who received enzastaurin/R-CHOP compared with patients who received R-CHOP alone, a benefit favoring enzastaurin $(p=0.018)$.

- These data suggest that addition of enzastaurin to R-CHOP may significantly improve outcomes as frontline therapy for high-risk DGM1+ DLBCL.

ENGINE study

- ENGINE is a global, multicenter, placebo-controlled, randomized study to compare the effect of $\mathrm{R}-\mathrm{CHOP} /$ enzastaurin versus $\mathrm{R}-\mathrm{CHOP} /$ placebo as frontline treatment in high-risk DLBCL patients.

- The primary end point for this study is OS in patients who are DGM1+. Secondary end points include CR, ORR (CR and PR) in patients who are DGM1+; OS in patients who are DGM1- and EFS including EFS12 and EFS24, for all the patients, and for the patients who are DGM1+. Eligibility criteria include naive-treatment, histologically confirmed CD20-positive DLBCL, high risk patient population IPI = 3, 4, 5 and DGM1+ or DGM1 -. Approximately 235 patients will be randomized 1:1 ratio to RCHOP/enzastaurin versus RCHOP/placebo stratified by IPI score (3 vs $4,5)$ and by region (USA vs China).

- DGM1 biomarker testing is performed in batches retrospectively after randomization and all patients will receive up to six cycles.

- PET-computer tomography will be used for staging and to assess radiographic response at the end of combination phase treatment.

- Patients randomized to the enzastaurin arm who achieve CR or PR (at investigator's discretion) will have the opportunity to continue in the single-agent phase of the study and receive single-agent enzastaurin for up to 2 additional years.

- The study has a $90 \%$ power to detect a HR of 0.45 .

- The study is currently recruiting with expected accrual completion in June 2020 and more information is available at ClinicalTrials.gov (NCT03263026).

\section{References}

Papers of special note have been highlighted as: $\bullet$ of interest; $\bullet \bullet$ of considerable interest

1. WHO Classification of Tumours of Haematopoietic and Lymphoid Tissues. Swerdlow SH, Campo E, Harris NL, Pileri S, Stein H, Elaine S. (Eds). IARC Press, Lyon, France (2008).

2. Møller MB, Pedersen NT, Christensen BE. Diffuse large B-cell lymphoma: clinical implications of extranodal versus nodal presentation - a population-based study of 1575 cases. Br. J. Haematol. 124, 151-159 (2004).

3. Intragumtornchai T, Bunworasate U, Wudhikarn K et al. Non-Hodgkin lymphoma in South East Asia: an analysis of the histopathology, clinical features and survival from Thailand. Hematol. Oncol. 36 (1), 28-36 (2018).

4. Nowakowski G, Chiapella A, Witzig T et al. ROBUST: lenalidomide-R-CHOP versus placebo-R-CHOP in previously untreated ABC-type diffuse large B-cell lymphoma. Future Oncology 12(13), 1553-1563 (2016).

5. Roschewski M, Dunleavy K, Wilson WH. Diffuse large B-cell lymphoma: molecular targeted therapy. Int. J. Hematol. 96(5), 552-561 (2012).

-• Elegant review of diffuse large B-cell lymphoma (DLBCL) associated molecular pathways and potential treatment strategies. 
6. Sehn L, Gascoyne R. Diffuse large B-cell lymphoma: optimizing outcome in the context of clinical and biologic heterogeneity. Blood 125, 22-32 (2015).

- Interesting lecture of DLBCL therapies from combination of rituximab, cyclophosphamide, doxorubicin, vincristine and prednisone (R-CHOP) to novel therapies as enzastaurin in management of DLBCL

7. Zhou Z, Sehn LH, Rademaker AW et al. An enhanced international prognostic index (NCCN-IPI) for patients with diffuse large B-cell lymphoma treated in the rituximab era. Blood 123, 837-842 (2014).

8. Chiapella A, Castellino A, Vitolo U. State-of-the-art therapy for advanced-stage diffuse large B-cell lymphoma. Hematol. Oncol. Clin. North Am. 30, 1147-1162 (2016).

9. Maurer MJ, Ghesquières H, Jais JP et al. Event-free survival at 24 months is a robust end point for disease-related outcome in diffuse large B-cell lymphoma treated with immunochemotherapy. J. Clin. Oncol. 32(10), 1066-1073 (2014).

10. Robertson M, Kahl BS, Vose JM et al. Phase II study of enzastaurin, a protein kinase C beta inhibitor, in patients with relapsed or refractory diffuse large B-cell lymphoma. J. Clin. Oncol. 25(13), 1741-1746 (2007).

- Treatment with enzastaurin was well tolerated in previously treated DLBCL patients and associated with freedom from progression 20-50months after enrollment in the study.

11. Abramson J, Shipp M. Advances in the biology and therapy of diffuse large B-cell lymphoma: moving toward a molecularly targeted approach. Blood 106, 1164-1174 (2005).

12. Lee KW, Kim SG, Kim HP et al. Enzastaurin, a protein kinase C $\beta$ inhibitor, suppresses signaling through the ribosomal S6 kinase and bad pathways and induces apoptosis in human gastric cancer cells. Cancer Res. 68(6), 1916-1926 (2008).

13. Flowers CR, Sihna R, Vose JM. Improving outcomes for patients with diffuse large B-cell lymphoma. CA Cancer J. Clin. 60(6), 393-408 (2010).

14. Shipp MA, Ross KN, Tamayo P et al. Diffuse large B-cell lymphoma outcome prediction by gene expression profiling and supervised machine learning. Nat. Med. 8, 68-74 (2002).

15. Carducci MA, Musib L, Kies MS et al. Phase I dose escalation and pharmacokinetic study of enzastaurin, an oral protein kinase $\mathrm{C}$ beta inhibitor, in patients with advanced cancer. J. Clin. Oncol. 24(25), 4092-4099 (2006).

16. Kreisl T, Kotliarova S, Butman J et al. A Phase I/II trial of enzastaurin in patients with recurrent high-grade gliomas. Neuro Oncol. 12(2), 181-189 (2010).

17. Crump M, Leppa S, Habermann T et al. Randomized, double-blind, Phase III trial of enzastaurin versus placebo in patients achieving remission after first-line therapy for high-risk diffuse large B-cell lymphoma. J. Clin. Oncol. 34(21), 2484-2492 (2016).

-. Phase III study using enzastaurin as single agent as maintenance after R-CHOP show not significant improvement in disease-free survival in DLBCL patients with high risk of relapse.

18. Camicia R, Winkler HC, Hassa PO. Novel drug targets for personalized precision medicine in relapsed/refractory diffuse large B-cell lymphoma: a comprehensive review. Mol.Cancer 14, 207 (2015).

19. Bourhill T, Narendran A, Johnston R N et al. Enzastaurin: a lesson in drug development. Crit. Rev. Oncol. Hematol. 112, 72-79 (2017).

-. Review of the PKC as targeted therapy in oncology and the development and mechanism of action of enzastaurin.

20. Hainsworth JD, Arrowsmith ER, Fayad L et al. A randomized, Phase 2 study of R-CHOP plus enzastaurin vs R-CHOP in patients with intermediate or high-risk diffuse large B-cell lymphoma. Leuk. Lymphoma 57(1), 216-218 (2016).

-. Phase II study of RCHOP/enzastaurin showed prolonged progression-free survival and overall survival when administered as frontline therapy in DLBCL patients.

21. Li X, Fang X, Lin T et al. A pharmacokinetic and safety study of a fixed oral dose of enzastaurin $\mathrm{HCl}$ in native Chinese patients with refractory solid tumors and lymphoma. Oncotarget 7(14), 18585-18593 (2016).

22. Denovo Biomarker Platform Patent, Inc.: WO 2012/106267 (2012).

23. Luo W, Sun H, Shazer R et al. Improved survival with enzastaurin treatment in diffuse large B-cell lymphoma (DLBCL) patients with the novel genetic biomarker, DGM1. Presented at: American Society of Hematology. San Diego, CA, USA, 1-4 December 2018.

- Denovo genomic biomarker 1 is a predictive biomarker of enzastaurin efficacy.

24. Chen JQ, Bao Y, Lee J et al. Prognostic value of the trichorhinophalangeal syndrome-1 (TRPS-1), a GATA family transcription factor, in early-stage breast cancer. Ann. Oncol. 24(10), 2534-254 (2013).

25. Wu L, Wang Y, Liu Y et al. A central role for TRPS1 in the control of cell cycle and cancer development. Oncotarget 5, 7677-7690 (2014). 
